\title{
BIO.0I - Initial assays for the characterization of a biosimilar anti-PD-I monoclonal antibody to Nivolumab
}

Michael Bernardes Ramos ${ }^{1 *}$; Ana Paula Dinis Ano Bom; Renata Chagas Bastos; Anna Erika Vieira de Araujo ${ }^{1}$; Aline de Almeida Oliveira ${ }^{1}$; Patricia Cristina da Costa Neves ${ }^{1}$; Haroldo Cid da Silva Júnior ${ }^{1}$.

1Fiocruz/Bio-Manguinhos.

Introduction: According to the World Health Organization (WHO), cancer is the second leading cause of death in world's population. In Brazil, it's expected the occurrence of 600.000 new cases for year for the biennium 2018-2019. The immune system acts controlling and eradicating the disease. However, in malignancy scenario, several mechanisms of immune suppression may be present, reducing the antitumor immunity of the host. Thus, antibody therapy against immunological checkpoints, such as programmed cell death 1 (PD-1), has been shown to be successful in reversing immunosuppression. Biosimilars are biological medicines that have the same safety and efficacy as reference medicines. The substitution of reference products for biosimilars may increase patients' access to these drugs as well as reduce public health expenditures.

Objective: Characterize a biosimilar anti-PD-1 monoclonal antibody to Nivolumab (OPDIVO ${ }^{\mathrm{Tm}}$ )

Methodology: Anti-PD-1 antibody has been obtained by recombinant DNA technology using the Expi293F expression system. After its purification, the physico-chemical characterization has been continued using the fluorimetry, circular dichroism, isoelectric focusing and size exclusion chromatography assays. ELISA and Western blotting assessed binding capacity to the target. In addition, flow cytometry assays were performed to validate their binding to the PD-1 receptor anchored in the cell membrane. The reference antibody was subjected to the same assays to allow comparability parameters.

Results: At fluorescence spectra, the maximum peak observed was approximately $342 \mathrm{~nm}$ for both, suggesting that there are no drastic conformational differences between the antibodies and that conformation can be considered as native/enovelada. Circular dichroism spectra suggest that both have the same content and have the same type of secondary structure in beta sheet. The main electrophoretic band detected in the isoelectric focusing showed a pI value of 8.61 for the two antibodies. Chromatographic profile's observed in size exclusion was very similar, a main peak and two other peaks were observed which may be associated with the presence of aggregates in both. Anti-PD-1 bound specifically to the target receptor adsorbed on plate, exhibiting $\mathrm{OD}$ values for PD-1 receptor binding similar to the values observed to Nivolumab. In addition, using Anti-PD-1 antibody as the detection antibody for PD1 adsorbed on nitrocellulose membrane, a band of approximately $37 \mathrm{kDa}$ was observed in western blotting, suggesting that receptor recognition by the antibody may not depend on conformation. Finally, it was visualized that the biosimilar antibody recognizes and binds to the PD-1 receptor anchored in the cell membrane similarly to Nivolumab.

Conclusion: These results demonstrated that the biosimilar antibody obtained had physicochemical and target binding characteristics similar to the reference antibody. To assure the functional similarity of the Anti-PD-1 antibody to Nivolumab, cellular and biological activity assays will be performed in vivo.

Keywords: Anti-PD-1 antibody; Biosimilar; Nivolumab 